\title{
ON CONTRACTIVE MAPPINGS
}

\author{
MICHAEL A. GERAGHTY
}

ABstract. The Cauchy condition for convergence of a contractive iteration in a complete metric space is replaced by an equivalent functional condition. Many generalizations of the Banach-Neumann contractive mapping principle follow.

Introduction. Let $X$ be a complete metric space and let $f: X \rightarrow X$ be a contractive mapping or contraction, i.e.,

$$
d(f(x), f(y))<d(x, y) \text { for all } x, y \text { in } X .
$$

Let $x_{0}$ be a chosen point in $X$ and set $x_{n}=f\left(x_{n-1}\right)$ for $n>0$. Criteria for the sequence of iterates $\left\{x_{n}\right\}$ to be Cauchy are then of interest, for if it is Cauchy one can easily prove it converges to a unique fixed point $x_{\infty}$ of the $\operatorname{map} f$ on $X$.

Many papers have presented such criteria, especially since the important paper of Rakotch [1]. In this note we first give in $\S 1$ a necessary and sufficient condition that a sequence of iterates be convergent. This condition is used in $\$ 2$ to provide a criterion for the convergence of the iteration from an arbitrary initial point in $X$. The condition is then restated in a more familiar functional form, and some corollaries are then drawn in $\S 3$.

1. Main theorems. The following results, while stated for complete metric spaces, are readily extended to separated complete uniform spaces, as in [2, p. 197].

We shall use the following notation. For any pair of sequences $x_{n}$ and $y_{n}$ in $X$ with $x_{n} \neq y_{n}$, we write

$$
d_{n}=d\left(x_{n}, y_{n}\right) \text { and } \Delta_{n}=d\left(f\left(x_{n}\right), f\left(y_{n}\right)\right) / d_{n} .
$$

We then have the following theorem.

THEOREM 1.1. Let $X$ be a complete metric space. Let $f: X \rightarrow X$ with $d(f(x), f(y))<d(x, y)$, for all $x, y$ in $X$. Let $x_{0} \in X$ and set $x_{n}=f\left(x_{n-1}\right)$ for $n>0$. Then $x_{n} \rightarrow x_{\infty}$ in $X$, with $x_{\infty}$ a unique fixed point of $f$, iff for any

Received by the editors January 2, 1973.

AMS (MOS) subject classifications (1970). Primary 54H25; Secondary 47H10.

Key words and phrases. Contractive mapping, fixed points, convergence of iterations.

(c) American Mathematical Society 1973 
two subsequences $x_{h_{n}}$ and $x_{k_{n}}$ with $x_{h_{n}} \neq x_{k_{n}}$, we have that

$$
\Delta_{n} \rightarrow 1 \text { only if } d_{n} \rightarrow 0 .
$$

PRoof. First assume that $x_{n} \rightarrow x_{\infty}$ in $X$ and let $x_{h_{n}}$ and $x_{k_{n}}$ be any two subsequences. Then clearly $d_{n}=d\left(x_{h_{n}}, x_{k_{n}}\right) \rightarrow 0$ and so the condition is satisfied.

Next assume the condition is satisfied for a given initial point $x_{0}$ in $X$. Then $d_{n}=d\left(x_{n}, x_{n+1}\right)$ is a decreasing sequence of nonnegative numbers and so approaches some $\varepsilon \geqq 0$. Assume $\varepsilon>0$. Then letting $h_{n}=n$ and $k_{n}=n+1$, we have $d_{n} \rightarrow \varepsilon>0$ while $\Delta_{n} \rightarrow 1$. So the condition is violated. Thus $d\left(x_{n}, x_{n+1}\right) \rightarrow 0$.

Now assume the given sequence of iterates $\left\{x_{n}\right\}$ is not Cauchy. Then there exists some $\varepsilon>0$ such that every tail $\left\{x_{n}\right\}_{n \geq N}$ of the sequence has diameter $D_{N}=\sup _{n, m \geqq N} d\left(x_{n}, x_{m}\right)>\varepsilon$. Given this $\varepsilon$, we will construct a pair of subsequences violating the condition.

For any $n>0$, let $N_{n}$ be so large that $d\left(x_{m}, x_{m+1}\right)<1 / n$ for all $m \geqq N_{n}$, as is possible since $d\left(x_{m}, x_{m+1}\right) \rightarrow 0$. Let $h_{n} \geqq N_{n}$ be the lowest integer such that for some $k_{n}>h_{n}, d\left(x_{h_{n}}, x_{k_{n}}\right)>\varepsilon$. Such pairs exist by the above diameter condition. Next choose $k_{n}$ to be the least such integer above $h_{n}$. Then either $k_{n}-1=h_{n}$ or else $d\left(x_{h_{n}}, x_{k_{n}-1}\right) \leqq \varepsilon$. In either case we have $\varepsilon \leqq d_{n}=$ $d\left(x_{h_{n}}, x_{k_{n}}\right)<\varepsilon+1 / n$.

Moreover, using the triangular inequality on the contraction, we have

$$
1 \geqq \Delta_{n}=\frac{d\left(f\left(x_{h_{n}}\right), f\left(x_{k_{n}}\right)\right)}{d_{n}} \geqq \frac{d_{n}-2 / n}{d_{n}} .
$$

So $\Delta_{n} \rightarrow 1$ while $d_{n} \rightarrow \varepsilon>0$, again violating the condition.

So $\left\{x_{n}\right\}$ must be a Cauchy sequence in $X$ and as $X$ is complete, we have $x_{n} \rightarrow x_{\infty}$ for some $x_{\infty}$ in $X$. Then by the usual arguments, $x_{\infty}$ is a unique fixed point of $f$ and the proof is complete.

The above proof is essentially that of Theorem 6.1 in [2, p. 197] with the added observation that on a particular iteration, the condition is necessary. A close inspection of the proof shows that the following slight improvement was really proven.

Corollary 1.2. Let $X$ be a complete metric space. Let $f: X \rightarrow X$ with $d(f(x), f(y))<d(x, y)$ for all $x, y$ in $X$. Let $x_{0} \in X$ and set $x_{n}=f\left(x_{n-1}\right)$ for $n>0$. Then $x_{n} \rightarrow x_{\infty}$ in $X$, with $x_{\infty}$ a unique fixed point of $f$, iff for any two subsequences $x_{h_{n}}$ and $x_{k_{n}}$ with $x_{h_{n}} \neq x_{k_{n}}$, we have that $\Delta_{n} \rightarrow 1$, with $d_{n}$ decreasing, only if $d_{n} \rightarrow 0$.

Proof. Same as Theorem 1.1. 
We now wish to convert this sequential condition to the more customary functional form. Following Rakotch [1], we define a class of test functions more general than his.

Definition. $S$ is the class of functions $\alpha: R^{+} \rightarrow[0,1)$ with

(i) $\boldsymbol{R}^{+}=\{t \in \boldsymbol{R} \mid t>0\}$,

(ii) $\alpha\left(t_{n}\right) \rightarrow 1$ implies $t_{n} \rightarrow 0$.

Notes. (1) We do not assume that $\alpha$ is continuous in any sense.

(2) We only require that if $\alpha$ gets near one, it does so only near zero.

(3) Using Corollary 1.2, we could replace property (ii) with (ii') $\alpha\left(t_{n}\right) \rightarrow 1$ with $t_{n}$ decreasing implies $t_{n} \rightarrow 0$.

THEOREM 1.3. Let $f: X \rightarrow X$ be a contraction on a complete metric space. Let $x_{0} \in X$ and set $x_{n}=f\left(x_{n-1}\right)$ for $n>0$. Then $x_{n} \rightarrow x_{\infty}$, where $x_{\infty}$ is a unique fixed point of $f$ in $X$, iff there exists an $\alpha$ in $S$ such that for all $n, m$,

$$
d\left(f\left(x_{n}\right), f\left(x_{m}\right)\right) \leqq \alpha\left(d\left(x_{n}, x_{m}\right)\right) \cdot d\left(x_{n}, x_{m}\right) .
$$

Proof. We need only show that the existence of such an $\alpha$ in $S$ is equivalent to the sequential condition of Theorem 1.1. First assume such an $\alpha$ exists. Let $x_{h_{n}}$ and $x_{k_{n}}$ be subsequences with $x_{h_{n}} \neq x_{k_{n}}$. Assume that $\Delta_{n} \rightarrow 1$. Then it follows from the above inequality that $\alpha\left(d\left(x_{h_{n}}, x_{k_{n}}\right)\right) \rightarrow 1$. But then since $\alpha \in S$, we have $d\left(x_{h_{n}}, x_{k_{n}}\right) \rightarrow 0$.

Next assume that the sequential condition holds. Define $\alpha: \boldsymbol{R}^{+} \rightarrow \boldsymbol{R}$ as follows:

$$
\alpha(t)=\sup \left\{\frac{d\left(f\left(x_{n}\right), f\left(x_{m}\right)\right)}{d\left(x_{n}, x_{m}\right)} \mid d\left(x_{n}, x_{m}\right) \geqq t\right\} .
$$

Since $f$ is a contraction, the quotients are all below 1 and so $\alpha$ is defined for all $t>0$ and $\alpha \leqq 1$. Now assume that $\alpha\left(t_{n}\right) \rightarrow 1$ for $t_{n} \in \boldsymbol{R}^{+}$. We may further assume without loss of generality that $1-1 / n<\alpha\left(t_{n}\right) \leqq 1$. We must show $t_{n} \rightarrow 0$. But $\alpha\left(t_{n}\right)$ is the above least upper bound. So there is for each $n>0$ a pair $x_{h_{n}}, x_{k_{n}}$ in $\left\{x_{n}\right\}$ with

and

$$
d\left(x_{h_{n}}, x_{k_{n}}\right) \geqq t_{n}
$$

$$
1-\frac{1}{n}<\frac{d\left(f\left(x_{h_{n}}\right), f\left(x_{k_{n}}\right)\right)}{d\left(x_{h_{n}}, x_{k_{n}}\right)} \leqq \alpha\left(t_{n}\right) .
$$

So the sequence $\Delta_{n} \rightarrow 1$. But then by the sequential condition of Theorem $1.1, d\left(x_{h_{n}}, x_{k_{n}}\right) \rightarrow 0$. So $t_{n} \rightarrow 0$, as was to be shown. This completes the theorem.

2. A general convergence criterion. We may now apply the results of $\S 1$ to obtain a criterion for convergence of the iteration from an arbitrary 
starting point. Unfortunately, some generality is lost and the universal condition, while still sufficient, is no longer necessary.

THEOREM 2.1. Let $f: X \rightarrow X$ be a contraction of a complete metric space satisfying

$$
d(f(x), f(y)) \leqq \alpha(d(x, y)) \cdot d(x, y)
$$

where $\alpha \in S$. Then for any choice of initial point $x_{0}$, the iteration $x_{n}=$ $f\left(x_{n-1}\right), n>0$, converges to the unique fixed point $x_{\infty}$ of $f$ in $X$.

Proof. Simply apply Theorem 1.3 to any initial point $x_{0}$.

It should be noted that Theorem 2.1 is essentially the same as Corollary 1 of [2, p. 197]. The improvement afforded by the above presentation is that the essential character of the condition on each orbit is brought out in $\S 1$.

3. Some corollaries. The usefulness of the contraction mapping principle has prompted many generalizations of the original result, which is Theorem 2.1 with $\alpha \in S$ being simply a constant function. A few of these are listed below, all of them following easily from Theorem 2.1 (which, as pointed out in $\S 2$, is really little more than Corollary 1 of [2, p. 197]).

COROllaRy 3.1 (RAKOTCH [1, p. 463]). If $f: X \rightarrow X$ is a contraction of a complete metric space satisfying $d(f(x), f(y)) \leqq \alpha(d(x, y)) \cdot d(x, y)$ where $\alpha: \boldsymbol{R}^{+} \rightarrow[0,1)$ and is monotone decreasing, then for any choice of $x_{0}$ in $X$, the iteration $x_{n}=f\left(x_{n-1}\right), n>0$, converges to a unique fixed point $x_{\infty}$ of $f$ in $X$.

Proof. Such an $\alpha$ is clearly in the class $S$.

COROLlaRY 3.2. If $f: X \rightarrow X$ is a contraction of a complete metric space satisfying $d(f(x), f(y)) \leqq \alpha(d(x, y)) \cdot d(x, y)$ where $\alpha: \boldsymbol{R}^{+} \rightarrow[0,1)$ and is monotone increasing, then for any choice of $x_{0}$ in $X$, the iteration $x_{n}=$ $f\left(x_{n-1}\right), n>0$, converges to a unique fixed point $x_{\infty}$ of $f$ in $X$.

Proof. As in Corollary 3.1.

Corollary 3.3 (Boyd-Wong [4, p. 331]). If $f: X \rightarrow X$ is a contraction of a complete metric space satisfying $d(f(x), f(y)) \leqq \alpha(d(x, y)) \cdot d(x, y)$ where $\alpha: \boldsymbol{R}^{+} \rightarrow[0,1)$ and is continuous, then for any choice of $x_{0}$ in $X$, the iteration $x_{n}=f\left(x_{n-1}\right), n>0$, converges to a unique fixed point $x_{\infty}$ of $f$ in $X$.

Proof. As in Corollary 3.1.

REMARK 1. The last corollary is derived from Theorem 1 of $[3$, p. 459]. This latter theorem also seems related to Theorem 2.1 and is a corollary, 
if we were to use the slightly stronger form provided by the Note (3) on the class of functions $S$ in $\S 1$.

REMARK 2. A paper investigating the relations among these various corollaries will shortly be submitted to the Journal for Mathematical Analysis and Applications.

\section{REFERENCES}

1. E. Rakotch, A note on contractive mappings, Proc. Amer. Math. Soc. 13 (1962), 459-465. MR 26 \#5555.

2. M. A. Geraghty, On Lipschitz structures, J. Math. Anal. Appl. 11 (1965), 191-198. MR 31 \#6211.

3. D. W. Boyd and J. S. W. Wong, On nonlinear contractions, Proc. Amer. Math. Soc. 20 (1969), 458-464. MR 39 \#916.

4. J. S. W. Wong, Mappings of contractive type on abstract spaces, J. Math. Anal. Appl. 37 (1972), 331-340. MR 45 \#1147.

Department of Mathematics, The University of Iowa, Iowa City, Iowa 52240 